\title{
The Analysis of a Blood Pressure Diary for a Patient Report
}

\author{
Gérard Näring ${ }^{1}$ and Cees van der Staak \\ University of Nijmegen
}

In order to make a more valid assessment of blood pressure (BP), self-monitoring of $B P$ by the patient is generally advised. Self-monitoring usually results in a lower $B P$ level. We are currently investigating whether the $B P$ lowering effect of self-monitoring may be enhanced by not only monitoring $B P$ but also monitoring physical and emotional status at the moment of and activity prior to the BP measurement in a diary. In this article we focus on the individual feedback that the participating patients received. Various methods that can be used for this purpose are described. For our individual reports we first assessed linear trends in the variables. When a trend was present, residuals were calculated. Then a principal-components analysis on $B P$ measurements, BP estimates, symptoms, moods, and activities was performed. Results are presented for two hypertensive patients.

Descriptor Key Words: blood pressure; self-monitoring; mood; symptoms.

Casual blood pressure (BP) readings by the general physician often do not give an accurate picture of actual BP (Battig, Steiner, Jeck, \& Vetter, 1989). Many people exhibit a rise in BP when confronted with a physician or nurse, a phenomenon called office hypertension or white coat hypertension. White coat hypertension may affect some $40 \%$ of hypertensive patients, for whom consequently too much or unnecessary medication is prescribed (Hall, Higgs, \& Notarianni, 1990). In order to make a more valid assess-

${ }^{1}$ Address all correspondence to Dr. G. Näring, Department of Clinical Psychology and Personality, University of Nijmegen, P.O. Box 9104, 6500 HE Nijmegen, The Netherlands; E-mail: naring@psych.kun.nl. 
ment of BP level, self-monitoring of BP by the patient at home is advised and might well become a standard diagnostic tool in general practice.

During a prolonged period of BP self-monitoring a substantial drop in BP is often seen over a period of up to 6 weeks (Laughlin, Fisher, \& Sherrard, 1979). By monitoring their BP daily, people understandably become aware of the differential influence of work, sleep, sports, and food on BP. Self-monitoring is therefore thought to contribute to changes in lifestyle that in turn cause a lowering of BP (Laughlin, Fisher, \& Sherrard, 1979). Self-monitoring of BP can thus be seen as a feedback procedure of which the BP lowering effect may be enhanced by not only monitoring BP but also monitoring physical and emotional status at the moment of BP measurement.

Monitoring symptoms and moods may furthermore be useful in teaching patients to more accurately estimate their BP. Pennebaker and his colleagues examined whether it is possible to teach people to estimate BP more accurately (Barr, Pennebaker, \& Watson, 1988). In a laboratory, the individual association of $\mathrm{BP}$ with various symptoms and moods was assessed and subsequently told to the normotensive participants. As a result, the subjects improved their ability to accurately estimate BP. A relationship between BP and physical symptoms was also found in hypertensives, although this relationship was only weak when antihypertensive medication was used (Pennebaker \& Watson, 1988).

We are currently investigating whether the use of a diary during a prolonged period of self-monitoring intensifies the documented BP lowering effect. Furthermore, an assessment of the relationship between BP and symptoms and moods in the laboratory will be compared to an assessment in the natural environment. Results of this study will be published elsewhere. In this article we will focus on the individual feedback that the participating patients received. Individual feedback requires an analysis that produces information that is relevant to the patient and easy to understand. In order to be applicable in general practice such an analysis should furthermore not be too complicated or time-consuming to prepare.

One of the first descriptions of a BP assessment procedure that results in a report to the patient is that by Pennebaker and his colleagues (Pennebaker, Gonder-Frederick, Stewart, Elfman, \& Skelton, 1982). In their laboratory assessment they asked the patient after each of a series of tasks and rest-periods to give a report of symptoms and moods. Blood pressure was repeatedly measured and correlations between the patient's self-reports and BP measures were calculated and told to the patient. Such a report provides the patient with a list of symptoms and moods that covary with BP. A disadvantage of such a procedure is, however, that the relationship between symptoms and moods is not investigated. At different moments 
different symptoms or subgroups of symptoms may covary with BP. Furthermore, the fact that we are dealing with repeated measures is statistically ignored.

Repeated observations on the same subject are likely to be serially dependent. Serial dependency of observations within variables can easily disturb the relationship between variables that have our interest and consequently has to be dealt with (Van Egeren, 1989). Serial dependency can manifest itself in several forms. When repeated measures during a laboratory experiment are taken to measure task-specific arousal, other variance components are minimized by experimental and statistical control (Turpin, 1989). During an experiment in a laboratory, physiological measures will usually change monotonically as a function of time. Linear, logarithmic, and negative exponential curve-fitting procedures are used to describe and measure these trends; sometimes they are fitted to the rest values of variables in-between tasks (Geenen, 1991). Others used a factor analytic approach to deal with time dependence (Hettema, 1989).

While much is known about the shape of trends in physiological measures during laboratory sessions, we know less about home-collected BP measurements, symptoms, and moods in an arbitrary six-week period. A drop in BP can, however, be expected and can, with our purpose in mind, be quite adequately described with a linear function. After assessment of a trend we will remove it statistically before continuing to analyze day-specific rises in BP. There is also a practical reason to investigate only the presence of a linear trend, as tests for linear regression and the subsequent calculation of residuals are available in common statistical software packages. Similar tests will also be applied to the symptoms, moods, and activities.

The next step is to determine the day-to-day relationships among symptoms, moods, activities, and BP. In within-subject studies on the influence of stress on recurrent headache (Mosley et al., 1991) and fibromyalgia (Hazlett \& Haynes, 1992) separate time-series regression analyses were employed. In the latter two studies the effect of one or a few predictors on symptom reporting was assessed. When, however, as in our case studies, a set of related variables is used, a single variable that best predicts $B P$ can suppress the clinical significance of other variables. Furthermore, the significant predictors that result from a regression analysis will not necessarily constitute a recognizable pattern of symptoms and moods to the patient. An individual patient report should preferably contain a cluster of related symptoms and moods that covary substantially with BP and not necessarily a set of symptoms and moods that significantly predict BP. Methods to achieve such an analysis are rarely documented. Hazlett and Haynes (1992) cite a study by Smolen in which factor analysis was performed on the self-ratings of a single fibromy- 
algia subject on 14 emotional states over a 35-day period. Increases in a factor Time Pressure preceded an exacerbation of pain. While Smolen's study tried to establish a causal relationship between stress and pain, we are interested in a constellation of moods and symptoms that does not necessarily give rise to a higher BP, but covaries with BP. Instead of assessing whether factor scores resulting from a factor analysis on symptoms and moods predict a higher BP, we will perform an analysis on symptoms, moods, activity, BP, and BP estimates simultaneously. In order to enable a comparison with the results of another method described, we will also report a regression analysis of BP on symptoms and moods. Results will be presented for two hypertensives.

\section{METHOD}

\section{Subjects}

Patient 1 was a 43-year-old man with a 9-year history of hypertension, whose BP was controlled by a $\beta$-blocker and a diuretic. Patient 2 was a 53-year-old man with a 10-year history of hypertension, who was not taking medication.

\section{Materials}

BP Measuring Device. Patients used a Takeda UA751, an automatic oscillometric device, that performs measurements automatically and provides prints of systolic BP, diastolic BP, heart rate and time of the measurement (Johnston \& Shah, 1989).

Diary. Patients received a booklet with instructions on how to use the $\mathrm{BP}$ measuring device and how to use the diary. A further 42 identical pages constituted the diary that was to be filled in once a day. On each page the patient was asked to fill in date, time of measurement, and estimate of BP. The presence or absence of several symptoms and moods was indicated by circling one of the alternatives: not at all (1), a little (2), somewhat (3), rather (4), or strongly (5). The degree to which physical or cognitive activity had taken place in the $15 \mathrm{~min}$ before measurement was indicated on a five-point scale, and finally two BP measurements, spaced 2 min apart, were written down. Only the second BP measurement was used for analysis.

List of Symptoms and Moods. As BP is influenced by a variety of regulating mechanisms (Guyton, 1986) and changes in BP are not logically linked to specific symptoms, we selected symptoms from each of the groups of physical symptoms described in the Hamilton Anxiety Scale (HAS) 
(Bech, Kastrup, \& Rafaelsen, 1986) excepting urogenital symptoms. The list of symptoms consisted of: tense muscles, warm body, fast pulse, shortness of breath, blush, cold hands, pounding heart, perspiring, and sweaty hands. The list of moods was based upon the lists used in the Pennebaker studies (Pennebaker, Gonder-Frederick, Stewart, Elfman, \& Skelton, 1982) and consisted of the following seven moods: excited, angry, rushed, energetic, happy, relaxed, and sad. Subjects received instructions to give scores on a scale from 0 , representing the absolute absence of a symptom or mood, to 5, the highest intensity they had ever experienced. Furthermore, subjects were encouraged to use the complete range of scores in order to report even minor fluctuations in symptoms and moods.

\section{Procedure}

Patients received a letter from their family physician in which their cooperation was asked for our study. Enclosed was another letter, in which we briefly informed them about the nature of our research and invited them to participate. Furthermore, a reply card and addressed envelope were enclosed.

Upon visiting the laboratory, patients were more extensively informed about the purpose of our research and what we expected from them. After written informed consent was obtained the patient practiced taking a BP measurement. We gave the patient instructions to measure at home in a sitting position in front of a table in a quiet room, preferably with no other people present. At home, the diary had to be filled in first. After proper fixation of the cuff on the left arm, the arm was to be positioned on the tabletop and a first measurement could be taken. After 2 min of quiet rest, a second measurement had to be performed. The World Hypertension League advises instructing patients to wait at least 5 min before taking a BP measurement at home (World Hypertension League, 1988). Keeping the $\log$ and waiting 2 min between measurements ensures that this rest period has elapsed before the second measurement. We emphasized that measurements had to be taken within an interval of 1 hour before or after a fixed time every day. Although a choice by the patient for morning measurements was accepted, we first asked the patient to think about a time in the afternoon or evening. Our rationale is that more variability in measures is to be expected in the afternoon or evening than directly after waking up.

When the diary was reviewed, an appointment was scheduled for 8 weeks later. Patients received 6 envelopes to return their diary pages every week to our laboratory. During the second appointment the patients received a written report consisting of a graph of the BP during the meas- 
urement period and a description of the factors that were related to BP or BP estimate. The same report was sent to the family physician of the patient.

\section{Data Reduction and Analysis}

To make different modalities comparable, all raw scores were converted to $z$-scores (Hettema, 1994). A minimum-variance criterion was applied by omitting from analysis variables with less than three scores deviating from an otherwise constant series. The second systolic home BP measurement was used in analysis. First, Pearson product moment correlations were computed between the number of days in study and BP, BP estimate, symptoms, moods, and activities. Correlations of .39 or higher are significant, $p \leq .01$, and indicate a linear trend. Trend was subsequently removed with a linear regression analysis. Then a stepwise regression analysis was performed with BP as the dependent variable and the symptoms, moods, and activities as predictors. Finally, principal-components analysis on symptoms, moods, activities, BP, and BP estimates was performed using the Kaiser Criterion with subsequent Varimax rotation. As there is no a priori reason to assume uncorrelated factors, an oblique solution is also presented and discussed. Only variables with a factor loading of .39 or greater, sharing $15 \%$ or more variance with the construct, will be used for interpretative purposes (Stevens, 1986, p 345). As a measure of the subject's accuracy of BP estimation the difference between BP estimation and BP was calculated. A regression analysis of this discrepancy score on the number of days in the study indicates whether the accuracy of BP estimation of a subject changed during the study.

\section{RESULTS}

\section{Case 1}

The diary was completed at 42 days. The following 11 variables displayed too little or no variance and were omitted from analysis: tense muscles, warm body, fast pulse, shortness of breath, blush, cold hands, perspiring, sweaty hands, excited, sad, and at ease. The remaining 9 variables were transformed to $z$-scores. Contrary to expectation, self-monitoring in this patient did not lead to a reduction in BP. A significant correlation with the number of days in study was only seen for the variable BP estimate, $r=-.33$, indicating that the patient gave successively lower BP estimates. 
The linear trend in the variable BP estimate was removed by performing regression analysis on the variable day and substituting the residuals in the data set for the original values.

A stepwise regression of BP on the new data set resulted in a single predictor, angry, $\beta=.35, R^{2}=.12, p=.02$. The principal-components analysis that was performed next is presented in Table I. The orthogonal and oblique solutions give similar results. Factor 1 in the orthogonal as well as in the oblique solution indicates that a higher BP estimate was given

Table I. Eigenvalues and Factor Loadings for PrincipalComponents Analysis on BP, BP Estimate, Moods, Symptoms, and Activities of Case 1

\begin{tabular}{|c|c|c|}
\hline Eigenvalue & Magnitude & $\begin{array}{l}\text { Variance } \\
\text { proportion }\end{array}$ \\
\hline 1 & 3.33 & 37.0 \\
\hline 2 & 1.55 & 17.3 \\
\hline 3 & 1.19 & 13.2 \\
\hline
\end{tabular}

Orthogonal transformation solution varimax

\begin{tabular}{lccc} 
& \multicolumn{3}{c}{ Factor } \\
\cline { 2 - 4 } \multicolumn{1}{c}{ Variable } & 1 & 2 & 3 \\
\hline Pounding heart & .59 & -.59 & .72 \\
Angry & & & \\
Rushed & .71 & .92 & \\
Energetic & & .68 & -.52 \\
Happy & & & -.51 \\
Physical activity & .56 & & \\
Cognitive activity & .75 & & .75 \\
BP estimate & .80 & & \\
BP & & &
\end{tabular}

Oblique solution pattern matrix

\begin{tabular}{|c|c|c|c|}
\hline \multirow[b]{2}{*}{ Variable } & \multicolumn{3}{|c|}{ Factor } \\
\hline & 1 & 2 & 3 \\
\hline Pounding heart & .52 & & -.52 \\
\hline Angry & & -.68 & \\
\hline Rushed & .76 & & \\
\hline Energetic & & & .94 \\
\hline Happy & & .46 & .70 \\
\hline Physical activity & .52 & .58 & \\
\hline Cognitive activity & .74 & & \\
\hline BP estimate & .79 & & \\
\hline $\mathrm{BP}$ & & -.74 & \\
\hline
\end{tabular}

Note: Only factor loadings of .39 or higher are displayed. 
when the patient felt his heart pound, and felt rushed, mostly after physical or cognitive effort. As BP does not load significantly on this factor this tendency was discussed as an inaccurate belief with the patient. The second factor in the orthogonal solution, similar to the third factor in the oblique solution, was not discussed with the patient because it had no significant relationship with either BP or BP estimate. The third factor in the orthogonal solution and the second in the oblique solution indicate that BP is usually higher when the patient feels angry and not happy. Frequently, little physical effort was undertaken before. As the BP estimate did not load significantly on this factor, the patient was told that he was apparently unaware of this relationship. The regression of the discrepancy score of BP estimate and BP on the number of days in study was significant, $B=-.16$, $\beta=-.34, R^{2}=.12, p=.03$, indicating that the patient gave progressively better BP estimates.

\section{Case 2}

The diary was completed at 42 days. The variables tense muscles, blush, cold hands, and sweaty hands displayed too little or no variance and were omitted from analysis. The remaining 16 variables were transformed to $z$-scores. Significant correlations with the number of days in study were seen for the variables perspiring, $r=-.44$, BP estimate, $r=-.69$, and $\mathrm{BP}$, $r=-.34$. In this patient a drop in BP as well as BP estimates could thus be observed during the period of self-monitoring. The patient could not indicate a reason for the trend in the symptom perspiring. The linear trend in the variables perspiring, BP, and BP estimate was removed by performing regression analyses on the variable day and substituting the residuals in the data set for the original values.

A stepwise regression of BP on the new data set resulted in the predictors $\mathrm{BP}$ estimate, $\beta=.46$, and warm, $\beta=.32, R^{2}=.42, p<.001$. The principal-components analysis is presented in Table II. As no oblique solution was obtained after 25 iterations, only the orthogonal solution is presented. Significant loadings of BP are seen on Factor 2 and Factor 3. Factor 2 is dominated by the physical symptoms warm and perspiring and has a moderate loading of the BP estimate. Factor 3 comprises the symptoms fast pulse, pounding heart, and the mood excited and has a high loading of the BP estimate. Factors 2 and 3 indicate that this patient gave quite accurate BP estimates. Both factors were discussed in terms of mental and physical states with the patient. Factors 1,4 , and 5 were not discussed with the patient because they had no significant relationship with either BP or BP estimate. 
Table II. Eigenvalues and Factor Loadings for Principal-Components Analysis on BP, BP Estimate, Moods, Symptoms, and Activities of Case 2

\begin{tabular}{|c|c|c|}
\hline Eigenvalue & Magnitude & $\begin{array}{l}\text { Variance } \\
\text { proportion }\end{array}$ \\
\hline 1 & 5.29 & 33.0 \\
\hline 2 & 2.09 & 13.1 \\
\hline 3 & 1.68 & 10.5 \\
\hline 4 & 1.23 & 7.7 \\
\hline 5 & 1.05 & 6.6 \\
\hline
\end{tabular}

Orthogonal transformation solution varimax

\begin{tabular}{|c|c|c|c|c|c|}
\hline \multirow[b]{2}{*}{ Variable } & \multicolumn{5}{|c|}{ Factor } \\
\hline & 1 & 2 & 3 & 4 & 5 \\
\hline Warm body & & .82 & & & \\
\hline Fast pulse & & & .78 & & \\
\hline Shortness of breath & & & & .70 & \\
\hline Pounding heart & & & .48 & .58 & \\
\hline Perspiring & & .83 & & & \\
\hline Excited & & & .60 & -.53 & \\
\hline Angry & .87 & & & & \\
\hline Rushed & & & & & .70 \\
\hline Energetic & & & & -.51 & .54 \\
\hline Happy & .87 & & & & \\
\hline At ease & .79 & & & & \\
\hline $\mathrm{Sad}$ & .57 & & & .40 & \\
\hline Physical activity & & & & & .82 \\
\hline Cognitive activity & .66 & & .42 & & \\
\hline BP estimate & & .39 & .72 & & \\
\hline $\mathrm{BP}$ & & .60 & .53 & & \\
\hline
\end{tabular}

Note: Only factor loadings of .39 or higher are displayed.

The regression of the discrepancy score of BP estimate and BP on the number of days in study was significant, $B=-.16, \beta=-.41, R^{2}=.17$, $p<.01$. The patient thus gave progressively better BP estimates.

\section{DISCUSSION}

After a period of recording self-monitored BP and a list of symptoms, moods, and activities in a diary, individual feedback was given to hypertensive patients. For this purpose we performed a trend analysis of the data, removed the trend, and then performed a principal-components analysis. The method constitutes a relatively quick way of assessing changes in the monitored variables and gives an adequate picture of the relation- 
ships between variables through clusters of BP, BP estimate and symptoms, moods, and activities. An advantage is that the patient is not focused on a single predicting variable as would result from a regression analysis. Instead a recognizable pattern of variables that covaries with $\mathrm{BP}$ is presented.

While we only tested for linear trend in the variables, the presence of trends of higher order cannot be precluded. Additional tests for higherorder trends require even more time for data analysis. Even in their present form, data-entry and statistical analysis require several hours. Such an investment of time is probably only possible when patients participate in a research program. A first step toward a more economic program would be the use of pocket computers in which the patient keeps his logs.

Taking one's own BP may initially cause stress, to which the patient habituates. Such an influence of the measurement procedure on the mood of our patients would probably be reflected in a trend in the variables "rushed" and "at ease." Such a trend was, however, absent in these variables in both patients. An influence of measurement-related stress on BP was minimized by asking the patient to measure BP twice. A second measurement ensures 2 minutes of rest in a seated position, and facilitates habituation.

Self-monitoring of behavior can tend to diminishing compliance (Chesney \& Ironson, 1989). In our study, however, we encountered few missing values. The high compliance can be attributed to several factors. First of all, our patients were well motivated, which was probably due to the introduction of our program by the family physician. Secondly, compliance was enhanced by the introduction of the expectation of accuracy checks. We asked the patients to send their logs to us every week and emphasized that a valid picture of the relationship sought could only be obtained when the $\log$ was kept every day.

Another problem in self-monitoring is "reactance": the effect that the act of self-monitoring changes the behavior under observation. Our withinsubject design does not permit statements about reactive changes in the logged variables. We did not, however, expect large effects because the reactive effect is reduced when more than one variable is tracked (Haynes $\&$ Cavior, 1977). The direction of reactance is largely determined by the valence of the self-recorded behavior (Ciminero, Nelson, \& Lipinski, 1977). Therefore, honesty in data recording was stressed, while moods and symptoms were not valenced as positive or negative. The only variable that was implicitly valenced was $\mathrm{BP}$, as patients probably wished to reduce $\mathrm{BP}$.

The sample size is not large enough to ensure reliable factors (Stevens, 1986, p. 345), but a similar objection applies to alternative methods of analysis. For a regression analysis, the number of predictors is too high in comparison with the number of cases. If one chooses to calculate 
correlations, the large number of correlations with a single variable would require adjusting the significance level. A way out would be to raise the number of observations, but asking patients to monitor their BP for a longer period would undoubtedly affect compliance. The alternative is to reduce the number of symptoms and moods. In practice, most patients already perform a selection by keeping the score on a number of symptoms or moods constant. Unfortunately, this does not reduce the number of symptoms and moods sufficiently for every patient. The optimal assessment of the individual-specific relationship between BP and symptoms, moods, and activities demands a substantial number of possibly relevant variables. For statistical and practical reasons some highly idiosyncratic symptoms will, however, have to be removed from our list.

A way out of this dilemma might be to ask the patient to indicate which symptoms or moods he/she considers most responsive. During a pilot study we noticed that this question does not necessarily reduce the number of symptoms. A legitimate selection can only be made after careful study of a larger number of subjects both with and without medication.

As hypertensives often hold rigid, inaccurate beliefs about symptoms and emotions that covary with BP (Baumann \& Leventhal 1985), it is useful to question the patient about such beliefs. When a patient strongly believes that a symptom or mood that is not on the list accompanies a higher BP, this symptom or mood should be added to the list. Inaccurate beliefs may be implicit, and may only come forward through the statistical analysis, but they may also be explicitly stated by the patient. It is important to gather data in order to validate or correct such a relationship and subsequently discuss possible consequences with the patient. Furthermore, it would be useful to add an objective measure of stress, as there may be an inverse association between subjective and objective measures of stress in hypertensives (Winkleby, Ragland, \& Syme, 1988). Hypertensives may not recognize the symptoms that could tell them that they are under stress. For these subjects a more fruitful approach may be to pay more attention to objective environmental signs of stress than to subjective internal signs.

\section{REFERENCES}

Barr, M., Pennebaker, J. W., \& Wạtson, D. (1988). Improving blood pressure estimation through internal and environmental feedback. Psychosomatic Medicine, 50, 37-45.

Battig, B., Steiner, A., Jeck, T., \& Vetter, W. (1989). Blood pressure self-measurement in normotensive and hypertensive patients. Journal of Hypertension, 7 (Suppl 3), 59-63.

Bech, P., Kastrup, M., \& Rafaelsen, O. J. (1986). Minicompendium of Rating Scales for States of Anxiety, Depression, Mania, Schizophrenia with Corresponding DSM-III Syndromes. Copenhagen: Munksgaard. 
Baumann, L. J., \& Leventhal, H. (1985). "I can tell when my blood pressure is up, can't I?" Health Psychology, 4, 203-218.

Chesney, M. A., \& Ironson, G. H. (1989). Diaries in ambulatory monitoring. In N. Schneidermann, S. M. Weiss, \& P. Kaufmann (Eds.), Handbook of Research Methods in Cardiovascular Behavioral Medicine. New York: Plenum Press.

Ciminero, A. R., Nelson, R. O., \& Lipinski, D. P. (1977). Self-monitoring procedures. In A. R. Ciminero, K. S. Calhoun, \& H. E. Adams (Eds.), Handbook of Behavioral Assessment. New York: Wiley.

Geenen, R. (1991). Psychophysiological Consistency and Personality. Tilburg, The Netherlands: Tilburg University Press.

Guyton, A. C. (1986). Textbook of Medical Physiology. Seventh Ed. Philadelphia: Saunders.

Hall, C. L., Higgs, C. M., \& Notarianni, L. (1990). Home blood pressure recording in mild hypertension: Value of distinguishing sustained from clinic hypertension and effect on diagnosis and treatment. Journal of Human Hypertension, 4, 501-507.

Haynes, S. C., \& Cavior, N. (1977). Multiple tracking and the reactivity of self-monitoring: I. Negative behaviors. Behavior Therapy, 8, 819-831.

Hazlett, R. L., \& Haynes, S. N. (1992). Fibromyalgia: A time series analysis of the stressor-physical symptom association. Journal of Behavioral Medicine, 15, 541-558.

Hettema, J. (1989). Predictive validity. In J. Hettema (Ed.), Personality and Environment: Assessment of Human Adaptation. Chichester: Wiley.

Hettema, J. (1994). Psychophysiological assessment of personality using films as stimuli. Personality and Individual Differences, 16, 167-178.

Johnston, D. W., \& Shah, D. (1989). An evaluation of the Takeda UA751 automatic sphygmomanometer. Behavior Research and Therapy, 27, 203-204.

Laughlin, K. D., Fisher, L., \& Sherrard, D. J. (1979). Blood pressure reductions during self-recording of home blood pressure. American Heart Journal, 98, 629-634.

Mosley, H. T., Penzien, D. B., Johnson, C. A., Brantley, P. J., Wittrock, D. A., Andrew, M. E., \& Payne, T. J. (1991). Time-series analysis of stress and headache. Cephalalgia, 11, 306-307.

Pennebaker, J. W., \& Watson, D. (1988). Blood pressure estimation and beliefs among normotensives and hypertensives. Health Psychology, 7, 309-328.

Pennebaker, J. W., Gonder-Frederick, L., Stewart, H., Elfman, L., \& Skelton, J. A. (1982). Physical symptoms associated with blood pressure. Psychophysiology, 19, 201-210.

Stevens, J. (1986). Applied Multivariate Statistics for the Social Sciences. Hillsdale, NJ: Lawrence Erlbaum.

Turpin, G. (1989). Handbook of Clinical Psychophysiology. Chichester: Wiley.

Van Egeren, L. (1989). The analysis of continuous data. In N. Schneiderman, S. Weiss, \& P. Kaufmann (Eds.), Handbook of Research Methods in Cardiovascular Behavioral Medicine. New York: Plenum Press.

Winkleby, M. A., Ragland, D. R., \& Syme, S. L. (1988). Self-reported stressors and hypertension: Evidence of an inverse association. American Journal of Epidemiology, 127, 124-134.

World Hypertension League (1988). Self-measurement of blood pressure: A statement by the World Hypertension League. Journal of Hypertension, 6, 257-261. 